Ann. Abeille, I967, 10 (I), I7-27.

\title{
LES GLOBULARIACÉES VISITÉES PAR LES ABEILLES
}

\author{
Annemarie FOSSEL
}

Aigen-im-Ennstal, Autriche

SOMMAIRE,

Globularia cordifolia et Globularia mudicaulis qui sont considérées dans la littérature spécialisée comme des plantes visitées essentiellement par les Lépidoptères, le sont aussi par les abeilles dans les Alpes calcaires du nord, bien que les visites nécessitent souvent des vols à longue distance. Les pollens des deux espèces sont décrits pour la première fois de façon détaillée et l'aut eur attire l'attention sur la possibilité de les rencontrer dans les miels alpins.

\section{INTRODUCTION}

Depuis de nombreuses années je me suis efforcée de donner une description exacte des conditions de la miellée aux différentes altitudes de 1'Énnstal et, par extension, des Alpes d'Autriche. Mes travaux ont pour objet, d'une part de faire l'inventaire des plantes mellifères économiquement importantes et des producteurs de miellats, d'autre part d'examiner le spectre pollinique des miels récoltés, de façon à acquérir progressivement une meilleure connaissance des bases mellifères et des différents types de miels.

En observant les butineuses dans les champs et dans la campagne j'ai pu faire quelques constations intéressantes du point de vue de la biologie florale parmi lesquelles celles dont il sera question ici maintenant et qui concernent les visites faites par les abeilles à deax Globulaires alpines, Globularia cordifolia et G. nudicaulis.

\section{DISTRIBUTION ET ÉCOLOGIE}

La famille des Globulariacées est étroitement apparentée à celle des Scrofulariacées et a son centre de distribution dans le secteur méditerranéen. Le genre Globularia comprend en gros I 7 espèces dont je n'ai observé que deux, lesquelles appartiennent aux plantes de montagne du sud de l'Europe centrale. 


\section{a) Globularia cordifolia (Globulaire à feuilles en cœur)}

Son aire de répartition comprend : le Jura, le Massif central, les Alpes, l'ouest des Carpathes, les Apennins, l'Illyrie et le nord de la péninsule balkanique.

C'est un sous-arbrisseau gazonnant de 3 à Io $\mathrm{cm}$ de haut qui se propage fortement par des pousses épigées rampantes, ligneuses et radicantes mais qui ne prospère que sur un sol basique, riche en calcaire et pierreux.

Les prairies maigres et caillouteuses, les bancs rocheux ensoleillés, les pentes de graviers sèches, les bois clairs et les landes de bruyère conviennent particulièrement bien à la Globulaire à feuilles en coeur et on peut l'y trouver depuis le fond des vallées jusqu'aux alpages (2 $600 \mathrm{~m}$ ) en peuplements denses.

Les feuilles obtuses, bordées, en forme de cour et persistantes sont coriaces, toujours vertes et bien caractéristi(ques d'une espèce xérophile.

1)ans mon étroit secteur d'observation, l'Ennstal de Styrie, Globularia cordifolia est très répandue par endroits dans les Alpes calcaires depuis le massif de Dachstein à l'ouest jusqu'au 'Totengebirge au nord et aux Alpes d'Eisenerz à l'est. Sur les pentes abruptes exposées au sud dans les Alpes calcaires du nord ses coussins bas colonisent de préférence les sols pierreux, du fond des vallées jusqu'au-dessus de la limite des arbres en association avec Polygala chamaebuxus, Thymus serpyllum, Teucrium montanum, Helianthemum alpinum, etc. qui attirent aussi de nombreuses abeilles.

\section{b) Globularia nudicaulis (Globulaire à tige nue)}

Cette plante a son aire de répartition principale dans les Alpes calcaires du nord de la Suisse, de la Bavière et de l'Autriche mais elle se trouve aussi dans les Asturies, les Pyrénées et les Apennins, sur sol calcaire.

Les coussins très denses et très développés de ce sous-arbrisseau vivace de to à $30 \mathrm{~cm}$ de hauteur possèdent des feuilles entières obtuses, en forme de coin, d'aspect vernissé, coriaces, toujours vertes et qui ne sont pas consommées par le bétail.

Globularia nudicaulis préfère les prairies à Carex firma découvertes ainsi que les landes à Molinie bleue ou à Carex sempervirens qui sont caractéristiques des pâtures maigres alpines ou subalpines, sèches et riches en humus, ainsi que des pâturages caillouteux et des éboulis recouverts de végétation. Mais elle se trouve aussi dans les bois de Pins clairsemés, sous les Rhododendrons à côté d'Erica carnea et de Vaccinium entre 800 et $2600 \mathrm{~m}$.

Lans mon étroit secteur d'observation, les peuplements ayant une grande importance apicole, parce que très développés et très denses se trouvent dans la zone des alpages entre $\mathrm{I} 200$ et $\mathrm{x} 600 \mathrm{~m}$ où les pâturages du versant sud du Totengebirge se colorent en bleu-violet sur des kilomètres en juin et juillet; un mètre carré de coussin de Globulaire à tige nue peut porter jusqu'à I 5o inflorescences.

\section{I,ES FLEURS E'T I,A POLIINISATION}

Les fleurs des Globulaires sont groupées en inflorescences sphériques à l'extré. mité de tiges courtes dépourvues de feuilles ou portant seulement une ou deux folioles lancéolées. Ėlles ont un léger et agréable parfum de vanille, sont bleu-violet 

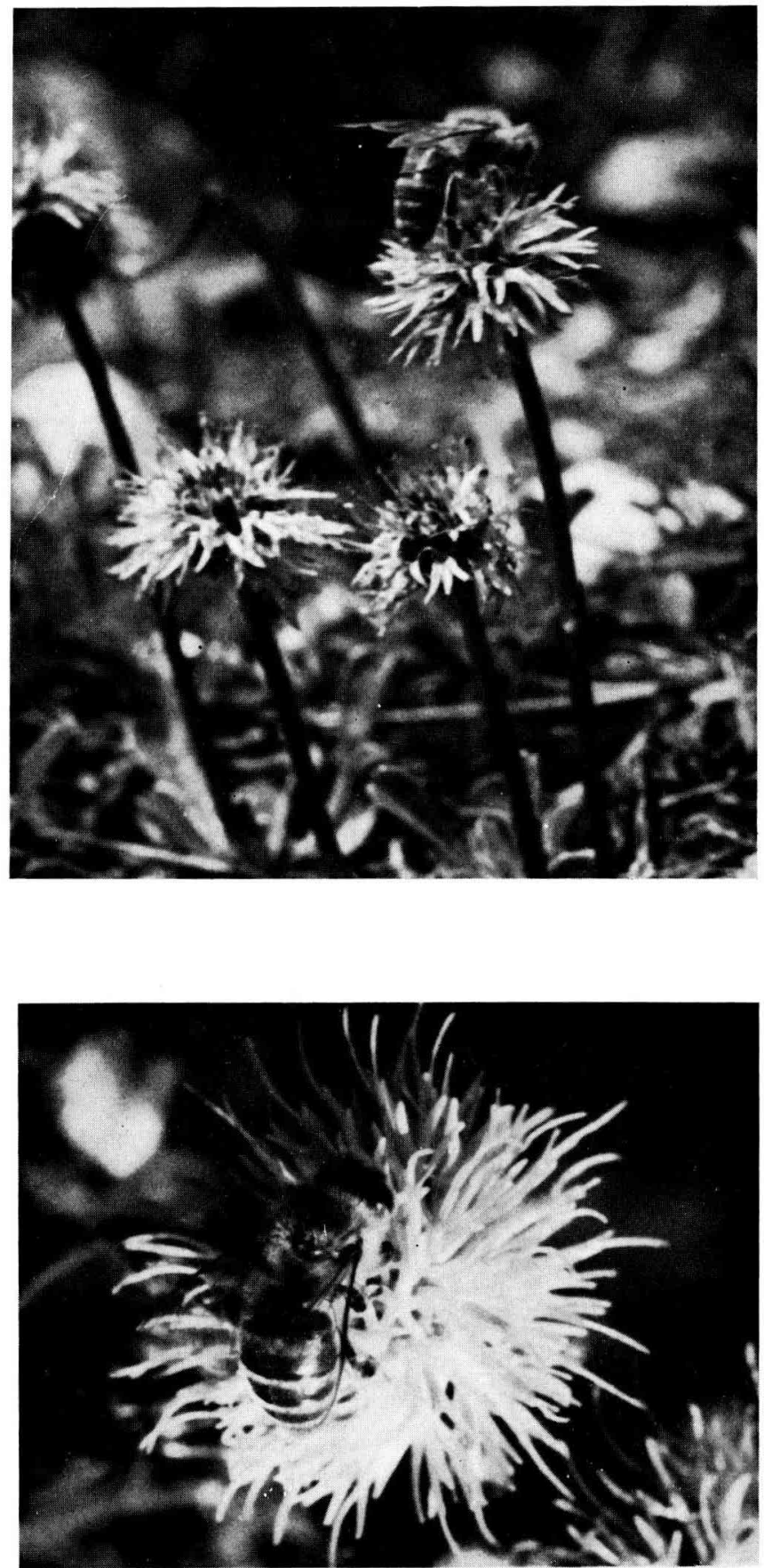

FI';. I. - En haut : alsille sur Globuluria cordifolia En bas : abeille sur (ilobularia nuticantis (Photo: A. losset) 
et mesurent chez $G$. nudicaulis $18-25 \mathrm{~mm}$, chez $G$. cordifolia Io-I5 $\mathrm{mm}$ de diamètre.

La corolle forme un tube à deux lèvres finement dentelées. Les quatre étamines sont soudées à la corolle et la dépassent largement. L'ovaire est supère et sécrète à sa base épaissie et charnue de telles quantités de nectar que celui-ci s'accumule dans l'étroite corolle.

Chez $G$. cordifolia la corolle a 6 à $\$ \mathrm{~mm}$ de longueur. La lèvre supérieure a deux lobes profonds; la lèvre inférieure porte trois lobes étroits. Chez G. nudicaulis la corolle étroite atteint une longueur de IO-I $2 \mathrm{~mm}$; la lèvre supérieure est atrophiée, la lèvre inférieure divisée en trois lobes linéaires.

La floraison commence en mai dans les fonds ; en altitude elle se produit en juin ou juillet, chez G. mdicaulis éventuellement encore en août. Toutes les inflorescences d'un même coussin ne s'ouvrent pas au même moment et les nombreuses fleurs de chaque tête s'ouvrent progressivement de l'extérieur vers l'intérieur, de telle sorte que le temps de floraison est relativement long.

Selon G. HEGr (I935) seuls les Iépidoptères sont à considérer comme pollinisateurs des Globulaires. In point de vue de la biologie florale il décrit les Globulariacées comme des plantes protérogynes lépidoptérophiles à fleurs bleues dont le nectar situé au fond d'une corolle profonde ne peut être commodément atteint que par la trompe des Papillons. Lorsque les visites cl'insectes font défaut il se produit, selon ses indications, une autofécondation facile et spontanée.

De son côté, E. ZANDER (r935) écrit que les Globulaires sont sans importance pour les abeilles. Il ne cite comme représentants du genre dans les montagnes de l'Allemagne que $G$. willkommii Hym. Malheureusement cette espèce manque dans mon secteur d'observation.

Selon les auteurs précédemment cités, le pollen des Globulaires attire différents insectes qui ne récoltent que le pollen libéré par les anthères qui dépassent largement de la corolle, ceci sans fonctionner comme pollinisateurs. Cependant, parmi les insectes récoltant dı pollen, l'abeille n'est pas citée dans la littérature.

\section{L'ABEILIE COMME POLLINISATEUR}

Selon des observations personnelles répétées et détaillées, l'Abeille est, dans 1'Ennstal de Styrie un visiteur fréquent et régulier de $G$. cordifolia et $G$. nudicaulis. Les abeilles abordent avec sûreté les inflorescences bleu lavande et introduisent leur tête énergiquement et profondément dans la corolle. Ellles visitent sur une inflorescence généralement plusieurs fleurs et travaillent activement : sans aucun doute elles atteignent le nectar et se polarisent sur cette source de nourriture.

Ce faisant, les butineuses se couvrent tout le corps d'un pollen blanchâtre que, cependant, elles ne brossent pas de leur pilosité. Fin altitude elles en font rarement des pelotes mais au contraire s'envolent couvertes de pollen vers l'inflorescence suivante dont elles assurent la pollinisation. Ce n'est qu'au voisinage des ruchers qu'on rencontre des butineuses de pollen sur les Globulaires et particulièrement $G$. cordifolia. Là, leur nombre est souvent important et les pelotes de pollen sont rondes et volumineuses. Lorsqu'on s'éloigne du rucher le nombre des butineuses de pollen diminue rapidement; elles finissent même par disparaître alors que sur Helianthe- 

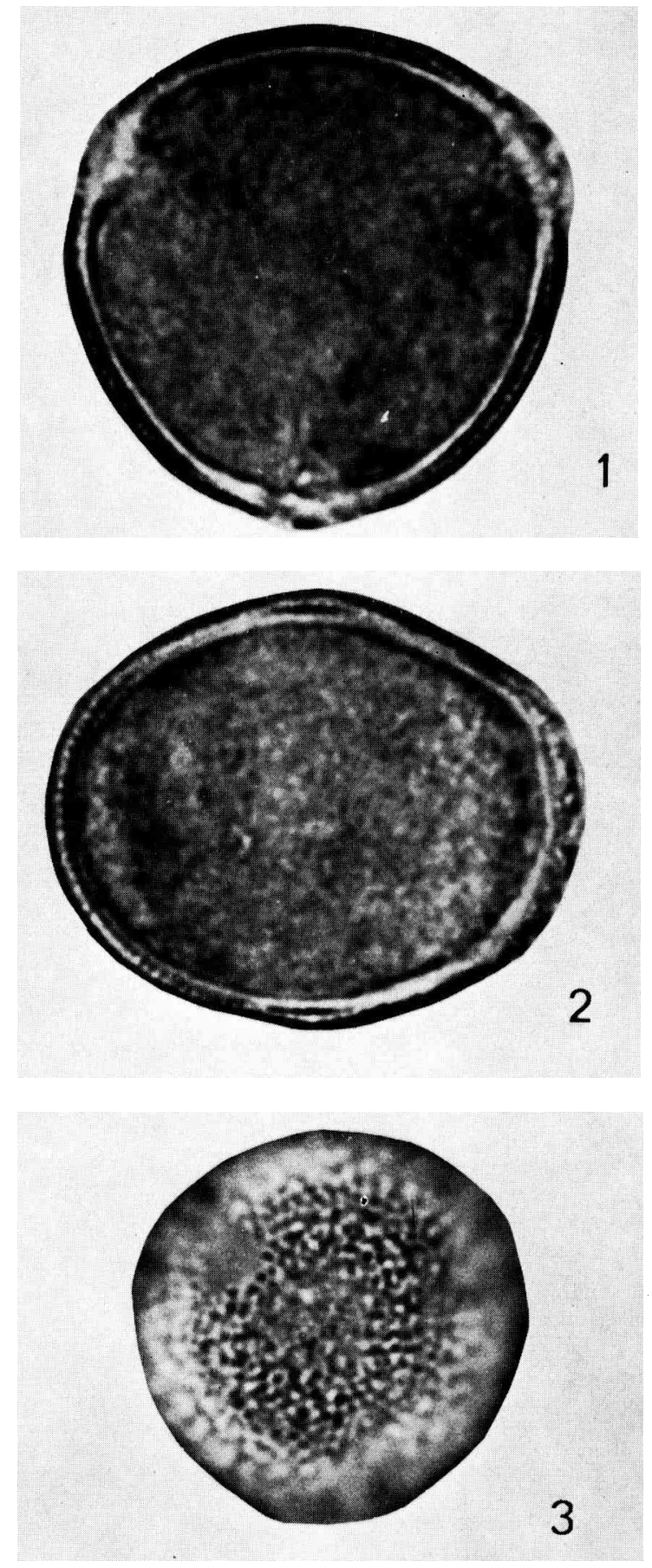

Plascie r. - Pollen de Globularia mudicaulis

I. Coupe optique équatoriale

2. Coupe optique mériclienne

3. Aire polaire 


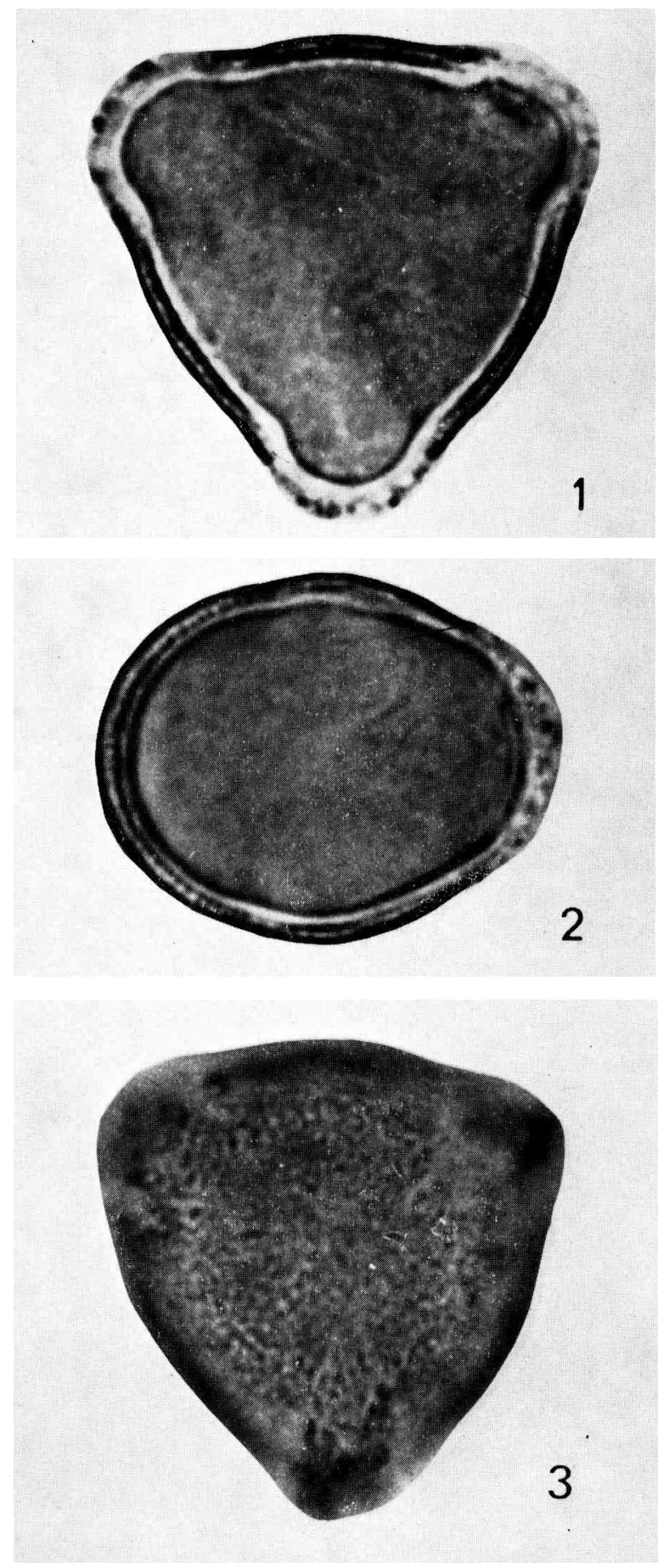

I'Lancies 2. - Pollen de Globularia cordifolia

I. Coupe optique équatoriale

2. Coupe optique méridienne

3. Nire polaire 

mum alpinum qui fleurit près des coussins de Globulaires, le pollen est toujours récolté activement.

Par contre, les butineuses de nectar, fait remarquable, se rencontrent encore sur les deux espèces de Globulaires $600 \mathrm{~m}$ plus haut alors que le rucher est éloigné de plus de I ooo m à vol d'oiseau.

En ce qui concerne Globularia cordifolia cette grande distance de vol est encore asse $z$ bien explicable. Les abeilles ont appris à récolter le nectar de cette plante au fond de la vallée, non loin des ruches, lorsque fin mai ou début juin les prairies ont été fauchées et que soudainement il a fallu trouver de nouvelles sources de nourriture. Une fois familiarisées avec ces fleurs les butineuses, recrutant en fonction de la miellée de nouvelles ouvrières, ont suivi toujours plus haut le long des éboulis abrupts les petites inflorescences bleues jusqu'à atteindre à l'été les bancs rocheux ensoleillés où fleurissent les derniers coussins de Globulaire à feuilles en cour.

En ce qui concerne le butinage de $G$. mudicaulis, actif sur les lointaines pâtures à I 200-I $600 \mathrm{~m}$ d'altitude, il n'existe pas d'autre explication que la valeur mellifère de la plante. A proximité des villages de montagne ou des fermes, là où se trouvent les ruchers, il n'existe pratiquement pas de Globulaire à tige nue, pas plus par exemple que de Rhododendrons qui, eux aussi sont découverts à partir de la vallée et activement visités.

La difficulté que représentent une différence de niveau de $600 \mathrm{~m}$ et une distance de plus de I ooo $m$ ne peut être surmontée par les butineuses de nectar, de façon régulière et en grand nombre, que si la source en vaut la peine. Il faut un grand nombre de fleurs d'une espèce donnée et il faut que ces fleurs fournissent un nectar abondant pour que le jabot puisse être rempli tapidement lorsque le but est atteint. En comparaison avec Rhododendron hirsutum, le butinage sur la Globulaire à tige nue est toutefois beaucoup moins intensif. On trouve sur une surface de Ioo $\mathrm{m}^{2}$ de Globulaire environ une butineuse contre ro et plus pour le Rhododendron.

L'examen du jabot des butineuses capturées sur $G$. cordifolia a généralement montré que celui-ci était rempli. Un contrôle dans les ruchers voisins a montré que par temps favorable beaucoup de butineuses rentrant à la ruche étaient poudrées d'un pollen blanc, ce qui est un signe caractéristique du butinage sur Globulaire.

\section{LE POLLEN : DESCRIP'TION E'T PRÉSENCE DANS LES MIELS}

Après montage en glycérine-gélatine selon la méthode préconisée par la Commission interna-

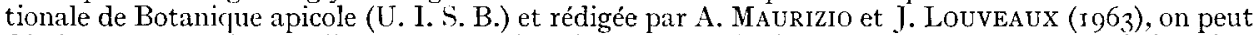
décrire comme suit le pollen de G. nudicaulis et $G$. cordifolia (échantillons provenant d'Aigen-imEnnstal) :

\section{Globularia nudicaulis (pl. I)}

Symétrie el forme : Grains isopolaires, tricolpés, subsphériques, subcirculaires à subtriangulaires en vue polaire.

Dimensions : $\mathrm{E}=26 \mu \quad \mathrm{P}=23 \mu$.

Apertures: Trois colpus à bords indistincts, de largeur maximum à l'équateur $\varepsilon=8 \mu$. Triangle polaire non mesurable avec précision mais probablement très petit. Nombreux petits fragments d'ectexine sur le sillon.

Exine : Épaisseur I $\mu$ environ. Endexine sensiblement de même épaisseur que l'ectexine. Présence d'épaississements polaires très nets. Columelles distinctes formant un réticulum très fin et uniforme. 
Intine : Mince et régulière.

Cytoplasme : Lisse à très finement granuleux.

Globularia cordifolia (pl. 2)

Symétrie et forme: Grains isopolaires, tricolpés, légèrement oblates, le plus souvent triangulaires en vue polaire, quelquefois subcirculaires.

Dimensions : $\mathrm{E}=24,5 \mu \mathrm{P}=18,5 \mu$.

Apertures: Trois colpus à bords assez peu distincts, légèrement saillants, de largeur maximum à l'équateur $\varepsilon=7 \mu$ environ. Triangle polaire rarement mesurable ; $t=6 \mu$ environ. Nombreux petits fragments d'ectexine sur les sillons.

Exine: Épaisseur a $\mu$ environ. Endexine sensiblement de même épaisseur que l'ectexine. Présence d'épaississements polaires très nets. Columelles distinctes formant un reticulum très fin et uniforme.

Intine : Mince et régulière.

Cytoplasme : Lisse à finement granuleux.

Il ressort de cette description et des planches photographiques correspondantes que le pollen des Globulariacées que nous avons étudiées est peu caractéristique, ce qui complique les identifications dans le culot de centrifugation des miels alpins. Lors d'un examen rapide des préparations microscopiques une confusion avec un pollen de Rosacée est presque inévitable. Même en recherchant systématiquement le pollen des Globulaires je n'ai pu le mettre en évidence qu'une fois comme pollen isolé. L'échantillon de miel provenait d'un rucher transhumant qui avait été installé à une altitude de I $500 \mathrm{~m}$ en $\mathrm{I}_{9} 6_{3}$ pour la miellée de Rhododendron. On récolta un miel monofloral de cette plante qui contenait de façon indiscutable quelques grains de pollen de Globulaire. Mais, lorsque le 20 juin les ruches arrivèrent au lieu de transhumance les Globulaires étaient presque défleuries. On peut toutefois penser que si les colonies avaient été transhumées plus tôt la miellée de Globularia aurait laissé une trace plus importante dans le spectre pollinique du miel.

De toute façon, les Globulaires à feuilles en coeur et les Globulaires à tige nue constituent une source de miellée dans les Alpes calcaires.

\section{CONCI,USION}

L'observation selon laquelle dans les Alpes calcaires d'Autriche Globularia mudicaulis et $G$. cordifolia sont visitées par les abeilles est intéressante non seulement du point de vue de la biologie florale mais aussi du point de vue apicole. Grâce à la construction de nouveaux chemins ruraux et forestiers la transhumance des abeilles en altitude se trouve facilitée. On peut y trouver, particulièrement en juin et juillet, d'abondantes sources de pollen et de nectar alors qu'au fond des vallées on constate souvent fin mai après les premières coupes un manque de pollen et une absence de miellée. Cette abondance en altitude est la garantie d'un bon développement des colonies et, si les conditions météorologiques sont favorables, de récoltes de miel satisfaisantes.

Rę̧u pour publication en janvier 1967.

\section{REMERCIEMEN'TS}

Je remercie M. Louveaux qui a bien voulu se charger de la description des pollens de Globularia nudicaulis et G. cordifolia ainsi que de leur photographie (planches I et 2). 


\section{SUMMARY}

GLOBE-DAISIES VISITED BY THE HONEYBEE

I have long been interested in the possibility of keeping bees high up in the mountains. The idea is quite sound, because these places have a natural carpet of flower-rich flora in an abundance unrivalled in Europe. I have found that two varieties of globe-daisiy ((Globularia nudicaulis and G. cordifolia) often occurring extensively on dry sites and lean meadows on the rocky terrain of the northern high Calcareous Alps are visited by the honeybee, despite the fact that they are described in the literature as lepidopter flowers. Honey-bees must be attracted to the pale blue heads of the bare-stalked and cardiophyllous globe-daisy which have a faint vanilla scent, since they often fly far and high above the tree-line, where spring does not come until July, to catch the last globedaisies in bloom.

Dr Louveaux has described and photographed the pollen forms of both globe-daisy varieties for the first time and it seems quite feasible that microscopic inspection reveals the presence of these pollens in the sedinient of honey from alpine flowers.

\section{ZUSAMMENFASSUNG}

DIE VON HONIGBLENEN BLILOGENE KLGLLBLUMEN

Schon seit Jahren bin ich bemüht, mir ein Bild über die bienenwirtschaftlichen Möglichkeiten. n höheren Gebirgslagen zu machen, da sich dort den Ionigbienen noch eine natürliche und blütenreiche Pflanzengesellschaft bietet, wie sic sonst in lituropa in solcher Vielfilt und Ausdehnung kaum mehr zu finden ist. Ich konnte beobachten, dass zwei Kugelblumenarten (Globularia nucaulis und $G$. cordifolia), die in den nördlichen Kalkalpen iuf felsigen Magermatten und trockenen Standorten oft ausgedehnte Bestände bilden, von den Honigbienen beflogen werden, obwohl die Blüten im einschlägigen Schrifttum als Falterblumen bezeichnet werden. I) Ie I Ionigbienen sammeln Pollen und Nektar und vermmit teln auch die Bestäiubung. Die blassblauen Blütenköpfchendernacktstengeligen und der herzb]ättrigen Kugelblume, die schwach nach Vanille duften, scheinen für die Honigbienen sehr anziehend zu scin, denn es werden oft weite Flugstrecken und grosse Höhenunterschiede überwunden, um hoch über der Baumgrenze, wo der Bergfrühling erst jm Juli Jinzug. hält, noch die letzten blühenden Kugelblumen zu erreichen.

Dr Louveaux beschreibt erstmals die Pollenformen der beiden Kugelblumenarten und zeigt Fotos, denn es wäre durchaus denkbar, dass bei der mikroskopischen lintersuchung von $\Lambda$ lpenblï: tenhonigen auch Kugelblumenpollen in Sediment gefunden werden.

\section{RÉFÉRENCES BIBLIOGRAPHIQUES}

HEGI G., I935. Illustriente Flora von Milleleuropa, Bul V1/1, 172-175, Hünich.

Matrizio A., Louvenux J., ig63. Méthodes d'analyse pollinique des miels. Ann. Abeille, 6 ( 1 ), 75-76. ZANDER E., I935. Beiträge zur Herkun/tsbeshimmumg bei Honig I. lollengestaltungr und IIerkunftsbestimung bei Blütenhonig, 287, Verlag der Reichsfachgruppe Imker, Berlin.

ZANDER E., I głr. Beiträge sur Ilerkunftsbestimmung bei Honig III. l'ollengrestaltung und I Ierkunftsbestimung bei Blïtenhonig, I00-Ior, Verlag Liedloff, L, th et Mlichaelis, Leipzig. 